\title{
Rache und Sprache
}

\author{
JULIANE PRADE-WEISS \\ Yale University \\ JENS KLENNER \\ Bowdoin College
}

\begin{abstract}
Rache und Sprache fügen sich in echohaftem Reim aneinander, und tatsächlich scheint die Rache in der Sprache wörtlich enthalten. Dennoch gibt es keine nachweisliche etymologische Verbindung zwischen den beiden Wörtern, wohl aber einen systematischen Zusammenhang: Sowohl in der Rede oder schriftlichen Kommunikation als auch in der Rachetat steht Wechselseitigkeit im Zentrum und ein Austausch soll etabliert werden. Die Sprache sucht nicht allein als Frage eine Antwort - in der Form einer These erheischt die Rede Erwiderung, als Klage verlangt sie Gehör, als Befehl fordert sie Gehorsam. Eine Rachetat sucht ebenso eine Erwiderungslogik zu etablieren, indem sie beansprucht auf eine Kränkung zu antworten und häufig eine Antwort in Form einer Gegenrache evoziert. Bereits zu Beginn des zwanzigsten Jahrhunderts sehen die Anthropologie und die Psychoanalyse die Wechselseitigkeit als strukturelle Analogie zwischen Sprache und Rache, wenn sie Klagen und Gewalt als zwei mögliche Antworten auf Todesfälle miteinander korellieren (Durkheim 658; Freud, GW IX 170-83). Aus verhaltenstheoretischer Sicht betonen jüngst Yoshimura und Boon, dass Rachehandlungen auf Austausch und Wechselseitigkeit bedacht sind: ,revenge is not simply a behavior, but rather is a communicated message" (Yoshimura/Boon 2).

Die Nähe von Sprache und Rache, die in der fundamentalen Rolle der Etablierung eines Austauschs liegt, betrifft freilich keineswegs allein deutschsprachige Gesellschaften und Literaturen. Doch zum einen macht der Reim von Rache und Sprache auf die Relation aufmerksam, die in anderen Sprachen weniger hervorsticht. Ähnlich phonetisch geht die russische Autorin Stepanova vor, wenn sie in einem Text von 2018 eine spezifische Präsenz der Rache in der deutschen Sprache und Erinnerungskultur hört: „Im deutschen Wort ,Erinnerung'schwingt für ein russisches Ohr ein ferner Anklang an die Erinnyen mit, die Rachegöttinnen, die den Schuldigen bis ans Ende der Welt vorfolgen“" (Stepanova 271-72). ${ }^{1}$ Zum anderen hat die Relation von Sprache und Rache als zwei Weisen der Etablierung von Wechselseitigkeit in deutsch-
\end{abstract}


sprachigen Debatten seit der Aufklärung besonderes Gewicht. Die Relation ist wichtig beispielsweise in der Diskussion der Rolle von Tränen im Menschen- und Männlichkeitsbild der griechischen Antike bei Winckelmann, Lessing und Herder (Lehmann 151; Richter 90-130; Weissberg 567-75). Diese und andere Diskurse über die Kodifikation von Affekten sind nicht zuletzt von der langwierigen Überführung des proto-juridischen Verständnisses der Rache im Fehderecht in ein psychologisches Verständnis individueller Innerlichkeit als Affekt oder Impuls motiviert.

Diese Überführung mündet im neunzehnten Jahrhundert in zwei alternative Ansichten über die Rache, die deren Schilderung nach wie vor bestimmen. Der Titel einer historischen Untersuchung des Völkerrechts von 2007 bringt sie auf den Punkt: Recht statt Rache oder Rache durch Recht? (Segesser). Die Position von „Recht statt Rache“ vertreten prominent die Gebrüder Grimm: Sie sehen in der Rache eine alte Rechtsauffassung gerechteneben gerächten - Handelns, die in der Neuzeit abqualifiziert wird zu einem „niedrige[n] Affekt“ (Schiller zit. in Grimm, Wörterbuch 14), gleichsam zum naming and blaming. Trotz dieser historiographischen Sicht auf Rachepraktiken wie „Buszen“ schreiben auch die Grimms mit am modernen Verständnis der Rache als Affekt von zeitloser Natürlichkeit:

[...] das natürliche gefühl nach empfangener beleidigung war rache, vergeltung, sühne; der flecken sollte getilgt und abgewaschen werden, dem beleidiger eine wenigstens gleich hohe oder höhere schmach widerfahren, es entsprang offene fehde und feindschaft (Rechtsalterthümer II, 646).

Verbreiteter als diese Sicht ist jene der Rechtsgeschichte, die Recht statt Rache setzt und sich dabei an der Orestie sowie an römischen Begriffen orientiert (Kaufmann 126-27; Probst/Sprenger II).

In diesem historischen Narrativ ersetzt nicht allein das Recht die Rache. Genauer betrachtet wird ein Substitutionsverhältnis von Rache und Sprache angenommen: Im kodifizierten Recht beendet der Urteilsspruch den Kreislauf von Rache und Gegenrache. Freuds Studien über Hysterie übernehmen 1898 dieses rechtshistorische Narrativ für die Ökonomie der Affekte, denn in der Sprache findet sich Freud zufolge ein „Surrogat“ für den „Racheakt“, dessen es bedarf, um eine Kränkung zu beantworten (GW1, 87). Zuschreibung, Aussage und Urteil erscheinen dabei jeweils als zugleich logisch-grammatische und juridische Mittel gegen die Ambivalenz aller Rache, stets Entsprechung und Restitution zu beanspruchen, aber proliferierende Destruktion zu bewirken. Beiden Ansichten von Rache ist es zu verdanken, dass die Rede von Rache selbst im juridischen Kontext ,in der heutigen Sprache von subjektivemotionalen Komponenten bestimmt" wird (Kaufmann 126). Es ist aber vor allem die Auffassung, das Recht habe die Rache ersetzt, die der archaischen Konnotation der Rache in der Gegenwart zugrundeliegt. Zur Faszination wie zur Abwertung genügt die Erwähnung von Rache, ,as if the very word 'revenge' offered sufficient evidence of the barbarism" (Jacoby 6). 
Gleichwohl hat die Rache als Struktur der Etablierung einer Logik der Wechselseitigkeit nichts an ihrer Relevanz eingebüßt. Derzeit scheinen internationale politische Beziehungen und innenpolitische Strategien auf beiden Seiten des Atlantiks von einer Logik der Vergeltung bestimmt. Die USamerikanisch geprägte Populärkultur bietet ein breites Spektrum von avengers und anderen „Rächern“, die eskapistisch oder kritisch das Versprechen auf ausgleichende Gerechtigkeit umsetzen, das trotz alledem in den meisten Lebensbereichen enttäuscht wird. Die Aussicht auf wechselseitigen Ausgleich wird, wie Wiggins argumentiert, dem kapitalistischen Text eingeschrieben: „Avengers 'get even' by settling accounts - financial and moral, personal and social - and recovering lost currency" (Wiggins 1). Und das deutsche Rabattkartensystem payback lockt namentlich damit, „Heimzahlung“ und ,Vergeltung" zu verschaffen. ${ }^{2}$

Trotzalledem wird die Zusicherung von Genugtuung durch Rache nicht überall als positiv gesehen. Die gegenwärtige Rechtstheorie und Moralphilosophie sieht die Vergeltungslogik der Rache nicht als Versprechen, sondern als Gefahr für individuelles wie politisches Miteinander und bemüht sich um eine Differenzierung zwischen Rache und Strafe (Zaibert 106-9; Cantarella 32-33). Die Unterscheidung erscheint umso notwendiger, als die altgriechische Terminologie, die mit ihrem historischen Paradigma demokratischer Institutionen nach wie vor als Referenzpunkt aufgerufen wird, „Rache“ und „Strafe“ im Wort timōria durchaus vereint (Probst/Sprenger I). Die Orestie sieht in der Buße fordernden Ausgleichslogik der Rache die Gefahr des Bürgerkrieges lauern (III 979-80), und ähnlich verstehen sich moderne Differenzierungen zwischen Rache und Strafe als dringend gebotene Trennungen zwischen Emotion und Institution. ${ }^{3}$ So plädiert Nussbaum nach einer Evokation des mörderischen Vergeltungskomplexes der Orestie gegen „Ärger“ und „Wut“" als Leitfaden des Handelns; nicht, weil es keinen Anlass zu Ärger und Wut gäbe, sondern weil sie auf direktem Wege zur Rache führten - ,the road of payback":

That road is normatively problematic because the beliefs involved are false and incoherent, ubiquitous though they are. They derive from deep-rooted but misleading ideas of cosmic balance, and from people's attempt to recover control in situations of helplessness (Nussbaum 5).

Nussbaum geht es um Normativität, daher fragt sie nicht nach Herkunft und Qualität des Glaubens an ,kosmische Balance“. Damit bleibt Nussbaums eröffnende Lektüre der Orestie, die sich auf die Verwandlung der Erinnyen in Eumeniden konzentriert, die Antwort auf die Frage schuldig, wie es möglich sei, dass die Erinnyen dem rachsüchtigen Glauben an Balance abschwören und Verhandlungen zugänglich werden - ,listen to the voice of persuasion" (Nussbaum 3). Das ist möglich, weil sowohl die Rache als auch die Sprache auf der Logik der Wechselseitigkeit beruhen, weil beide Erwiderung und Aus- 
tausch suchen. Und es ist just die nach Nussbaum „,fehlgeleitete“ Intention auf Gleichgewicht, die Wiggins zufolge die Lektüre populärer Rachenarrative lohnenswert macht: ,,payback plots [...] appraise the nation's political, social, or economic inequities" (Wiggins 11). Rächer sind somit, wie Kerrigan bemerkt, ,questers after psychic balance as well as ethical equivalence” (10).

Die Einsicht in die Intention auf Wechselseitigkeit in der Rache tritt jedoch im gegenwärtigen Diskurs der Sozial- und zunehmend auch der Geisteswissenschaften zurück hinter der Generalisierung der Rache als biologischer Konstante, deren angenommene prähistorische Herkunft sie einer Untersuchung entzieht. ${ }^{4}$,Revenge is probably the response that comes closest to our instinctual, biological reaction when faced with a wrong. [ . . ] [T] his archaic response has been handed down intact both in collective imagination and in social reality" (Speziale-Bagliacca 49, 18). Ähnlich referiert Groves Einsichten evolutionärer Psychologie:

$[\ldots]$ "strong responsiveness" $[\ldots]$ is a disposition to cooperate and punish those who violate social norms $[\ldots]$ Responsiveness $[\ldots]$ combines with rage to make up the complex emotion of revenge. [...] Emotions act "as if" certain things are true in the present moment, rightly or wrongly, simply because they were true in our ancestral past (Groves 24-25).

In diesen Schilderungen bieten sprachliche Strukturen Analogien zum Verständnis psychologischer und sozialer Zusammenhänge, wenn diese als „Antwort“, „Erwiderung“ und „Verantwortlichkeit“ beschrieben werden. Zugleich sollen aber die beschriebenen Strukturen Erklärungen zur Herausbildung etwa der Sprache bieten.

Gegen solches Übersehen der in Untersuchungen zur Rache ubiquitären Logik sprachlicher Wechselseitigkeit erhebt unser Themenheft Einspruch: Die Rache ist weder eine zeitlose biologische Konstante noch vorzeitlich und archaisch, sondern kulturell kodifiziert und kontextbestimmt. Im Altgriechischen wird das semantische Feld von Rache und Vergeltung je nach der genauen Organisation der Wechselseitigkeit noch mit mehr als zehn Begriffen beschrieben, zum Beispiel als dike (,Rechtsbrauch“), poine (,Blutgeld“), timōria (,Vergeltung“) oder geras (,Gabe“) (Descharmes 35-64). In gegenwärtigen westlichen Gesellschaften sind die dort entscheidenden Beziehungen eher fremd, und das der Blutrache zugrundeliegende relationale Ehrverständnis stellt eine Herausforderung für eine auf individueller Verantwortlichkeit basierende Jurisdiktion dar (Ermers 172-85). Kulturelle Kodifikationen von Rache sind zudem, wie jüngst Dawson und McHardy für die Literatur von der Antike bis zur englischen Renaissance schlaglichtartig zeigen, auch stark gender-konnotiert und fungieren in der attischen Tragödie etwa als Beweis für funktionale Männlichkeit und dysfunktionale Weiblichkeit (Dawson/McHardy 1-18). Die Rache ist, mit anderen Worten, ein soziales Phänomen, in deren Kodifikation sich Konventionen von Wechselseitigkeit 
und Austausch zuspitzen. Der Impuls zur Rache kann deshalb als kulturelle menschliche Konstante erscheinen, weil sie der sozialen Textur, das heißt dem Miteinander und Austausch, ebenso wesentlich angehört wie die Sprache. In einer politischen Gemeinschaft zu leben heißt für Menschen Aristoteles zufolge „Miteinandersprechendsein“ (Politik 1253a, Heidegger 47). Aber wie die in der Orestie vorgeführte Einsetzung öffentlicher Gerichtsbarkeit zeigt, liegt im Miteinander stets auch ein Gegen- und Füreinander-Rächen. Dabei werden Sprache und Rache in unterschiedliche Relationen zueinander gesetzt.

Die folgenden Essays konzentrieren sich auf Diskussionen des 19. und beginnenden 20. Jahrhunderts. Sie folgen der Annahme, dass die bürgerliche Kodierung des Affekthaushaltes den historischen Kontext darstellt, der gegenwärtige Diskussionen der Rache als biologisch oder archaisch, gefährlich oder lustvoll nach wie vor bestimmt: Diese Umcodierung transformiert den Katalog von Affekten, die zuvor als Hervorbringungen der Rhetorik verstanden wurden, in die Auffassung eines Inventars von Emotionen, die dem Menschen innewohnen und in der Sprache nicht fabriziert, sondern zum Ausdruck gebracht werden (Campe/Weber). Diese Naturalisierung der Affekte basiert auf der Erfindung des Begriffs „Gefühl“" um 1800 als neue Kategorie der „Darstellung von Erfahrung und Erleben“ (Lehmann 140-41; vgl. Greiner). Diese Umformung der Praxis des in Rhetorik und Musik verfertigten Affekts zum innerlichen Gefühlsleben als Quintessenz des Individuums erfasst auch das Verständnis der Rache: Führt Shakespeares Richard III noch die frühneuzeitliche Auffassung der Politik vor, dass ,,Machtgier und Rache die Substanz der Geschichte bilden“ (Ette 300), so erscheint die Relation von Rache und Gerechtigkeit in Nietzsches Zur Genealogie der Moral modernisiert als „,biologische[s] Problem“ (II.11, 310), dessen Verhandlung die Geschichte prägt. Ebenso schildern Texte des neunzehnten Jahrhunderts, wie Stifters in diesem Heft analysierte Erzählung ,Turmalin“ und am Beginn des zwanzigsten Jahrhunderts Freuds Studien über Hysterie die Rache als Tat, die aus einer Empfindung hervorgeht - und somit nicht mehr als historisch und kulturell kodifizierte Praxis von Recht oder Politik. Für die Diskussion im 21. Jahrhundert stellt die bürgerliche Umkodierung von Affekten zur Innerlichkeit im 19. Jahrhundert eine Schwierigkeit dar, denn die Naturalisierung lässt das „Gefühl“ als historisch kontingente biologische Konstante erscheinen (Lehmann 144), während sie in der Tat die historische Grundlage nicht allein für die Psychoanalyse und Psychologie legt, sondern ,die Bedingung der Möglichkeit noch der heutigen Emotionsformschung" ist (Lehmann 149). Darum bewegen sich auch die oben zitierten gegenwärtigen Schilderungen der Rache als archaisches Erbe aus der menschlichen Frühzeit in dem Paradigma, das im 19. Jahrhundert formuliert wurde.

Die Konzentration auf Austausch und Wechselseitigkeit macht über den historischen Rahmen hinaus auch auf eine systematische Schwierigkeit auf- 
merksam: Der Racheakt empfiehlt sich durch das Versprechen, einer Verletzung sichere Beachtung bei ihrem Urheber zu verschaffen. Jedoch kann er das Gehör und die Beherzigung, auf die es ihm zuallererst ankommt, ebenso wenig garantieren wie Flüche oder Verurteilungen. Umgekehrt kann die Sprache deswegen ein Surrogat oder Mittel der Rache sein, weil sie wesentliche Strukturen mit dieser gemein hat: die Intention auf eine Replik bewirkt eine Proliferation statt einer Wiedergutmachung. Die Erforschung der Erwiderungslogiken von Sprache und Rache im Forschungszusammenhang der in den Geistes-, Kultur- und Sozialwissenschaften seit einigen Jahren maßgeblichen, durchaus heterogenen Forschungsschwerpunkte der Affekttheorie und Emotionsgeschichte, geht jedoch in signifikanter Weise über sie hinaus. Während diese Ansätze die symbolische Verfassung bestimmter Empfindungen oder Gattungen betrachten, geht es in diesem Themenheft um die symbolische Organisation von Wechselseitigkeit, die sich in verschiedenen Diskursen erneuert und in unterschiedlichen Textsorten problematisiert wird. Im Zentrum stehen mithin weniger die historisch wie kulturell divergierende Kodifikation etwa von Wut, Zorn oder Eifersucht, sondern die Strukturen und Implikationen der sprachlichen oder praktischen Entgegnung, nach der sie je verlangen.

Die Schwierigkeit, Antwort und Erwiderung zu finden, die sich im Zusammenklang von Sprache und Rache zuspitzt, wird in Betrachtungen der Sprache in der kanonischen Philosophie oft zur Nebensache erklärt. In der Rhetorik jedoch ist sie ein wichtiger Teil der Systematisierung von Aufmerksamkeitsgewinn und Aufmerksamkeitslenkung, hier kanonisch in Aristoteles ${ }^{6}$ Abgrenzung des Aussagesatzes als einzig theoretisch relevanter Form der Rede von anderen Formen wie der euche - einer Bitte, einem Gebet, einem Schwur oder Fluch (Hermeneutik 16b-17a). Ziel dieses Themenheftes ist es, die Suche nach Gehör, Erwiderung und Antwort im sprachlichen Austausch sowie in der vermeintlichen Alternative gewaltsamen Handelns an kanonischen Werken zu präzisieren, sowohl um neue Lektüren dieser Texte zu erschließen, als auch um die Etablierung von Wechselseitigkeit als Komplikation auszuloten, die weitergehender Forschung bedarf.

Das Heft wird eröffnet mit Mona Körtes Beitrag Die Rache roher Texte. Treue zu Unsinn in den „Kinder- und Hausmärchen“ Jacob und Wilhelm Grimms. Diese Analyse des Erzählelements der Rache der Objektwelt in der Editionsgeschichte von Grimms Hausmärchen erlaubt ein vertieftes Verständnis der Transformation des Rachebegriffs, die sich im 19. Jahrhundert vollzieht. Der Beitrag schildert die immer weitergehende Kürzung und Streichung des Rachemotivs, das zunehmend als unverständlicher Fremdkörper innerhalb der Märchen erscheint. Dies ist darauf zurückzuführen, dass die Rache im zeitgenössischen Kontext der Edition nicht mehr als (obsolete) Praxis der Auseinandersetzung in Frage kommt, sondern allein als Empfindung. Der Objektwelt aber wird Innerlichkeit und somit auch die Möglichkeit zum Aus- 
tausch mit dem Menschen versagt. Mona Körtes Untersuchung steht damit im Zusammenhang mit gegenwärtigen Forschungslinien wie den „,material culture studies“ und den ,,animal studies“, in denen die Einschränkung von Subjektivität und Austauschmöglichkeit auf Menschen einer historischen und systematischen Kritik unterzogen wird. Die Kürzungen und anderen editorischen Eingriffe in die „Kinder- und Hausmärchen“, in deren Zug die Rache unbelebter Dinge gestrichen wird, geschehen zugunsten einer psychologischen Auffassung der Rache, die im Medium der Sprache ausgetragen wird. Einerseits ist es wesentlich die editorische Arbeit der Grimms, die einem psychologischen Racheverständnis zu Popularität verhilft, andererseits ist die ältere Auffassung von Rache als Rachetat, die in den Märchen mithin als unverständlicher Rest erscheint, gerade aufgrund des Erfolgs ihrer editorischen Arbeit in der Gegenwart ohne eine starke Kontextualisierung kaum noch erschließbar.

Umfassend formuliert wird das psychologische Verhältnis von Sprache und Rache in einem Text von Adalbert Stifter. Erica Weitzmans Despite Language: Adalbert Stifter's Revenge Fantasies ist ein close reading von Stifters Erzählung „Turmalin“, in der die Sprache Mittel einer Rache ist: Der im Stich gelassene Geliebte, dem die Erwiderung seiner Gefühle versagt wird, vergilt den Schmerz, indem er seiner Tochter die Möglichkeit sprachlichen Austauschs vorenthält. In dieser Erzählung hat Rache nichts mehr mit einer veralteten Rechtspraxis oder empfundener Gerechtigkeit gemein, sondern fällt als Skandalon und Faszinosum des bürgerlichen Zeitalters ganz in den Bereich des Gefühls und rätselhafter Innerlichkeit. Weitzmans Beitrag vermisst damit den Diskurs, in dem auch gegenwärtig Rachetaten und das Verständnis von Rache verhandelt werden, und an dem auch ältere oder andersartige Kodifikationen und Praktiken gemessen werden.

Der Beitrag von Juliane Prade-Weiss, Die Rache und ihr Surrogat. Zur Erwiderungslogik bei Freud, untersucht die Logik der Ersetzung in Freuds Deutungen der Rache und erlaubt damit ein vertieftes Verständnis der Psychologisierung der Rache. Dabei wird deutlich, dass Freuds psychoanalytische Theorie durchaus auf ältere Rechtsvorstellungen und antike Formen der Suche nach Wechselseitigkeit wie rituelle Klagen zurückgreift und sie in die moderne Form der Individualpsychologie übersetzt, in der die Möglichkeit des Austauschs mit anderen, von vermeintlicher ritueller Konventionalität befreit, zur Leitkomplikation der modernen Anforderung individueller Authentizität wird. Der Beitrag knüpft systemtisch an Körtes Analyse der Editionsgeschichte der Grimmschen „Kinder- und Hausmärchen“ an, insofern er aufzeigt, wie das scheinbar abseitige Thema der Rache zum zentralen Austragungsort von Narrativen der Modernisierung wird, die in juridischen, medizinischen, politischen und anderen Diskursen einhergehen mit einer grundsätzlichen Umstrukturierung gesellschaftlichen Austauschs und der Rolle des Individuums in Ökonomien von Wechselseitigkeit. 
Abgeschlossen wird das Heft von Tobias Heinrichs Beitrag Rache als Narrativ des Terrors. ,,Michael Kohlhaas“ und die Logik der Gewalt. Der Artikel untersucht den in deutschsprachigen Diskussionen der Rache wohl kanonischsten Text, Heinrich von Kleists „Michael Kohlhaas“, im Hinblick auf gegenwärtige Debatten über Terrorismus und Amok, für die Rache ein geläufiges Erklärungsmuster ist. Dass sich aber jegliches gewaltsame und unverständliche Verhalten als Rache bezeichnen lässt, kann als Resultat der in unserem Heft geschilderten, historisch und kulturell präzise bestimmbaren Kodifikation der Rache in der westlichen Moderne angesehen werden: Die moderne, psychologische Auffassung von Rache als empfindungsbasiert gewährt Verständlichkeit, indem sie das Unverständliche als archaisch und somit als Aufforderung zu einer korrigierenden, modernisierenden Antwort auffasst. Auch das ist eine Fehdeordnung.

${ }^{1}$ Die deutsche Fassung des Textes weicht an dieser Stelle vom russischen Original ab „auf Wunsch der Autorin“, wie der Verlag im Index erläutert.

${ }^{2}$ Vgl. https://www.payback.de/ [accessed 23. April 2019].

${ }^{3}$ An dieser Stelle ist von poinē (,Rache, Buße“) die Rede: di organ poinas antiphonous atas - ,,im Zorn der Rachgier wechselnden Mords Blutrausch“ (Übers. Werner).

${ }^{4} \mathrm{Vgl}$. Nussbaum, Anger and Forgiveness 39: "Anger, in short, has a very limited but real utility, which derives, very likely, from its evolutionary role as a 'fight-or-flight' mechanism."

\section{Zitierte Literatur}

Aischylos. Die Orestie. Übers. Email Staiger. Stuttgart: Reclam, 1958. Print.

Aristoteles. Hermeneutik. Übers. Hans Günter Zekl. Hamburg: Meiner, 1998. Print.

- Politik. Übers. Olof Gigon. Stuttgart: Reclam, ${ }^{2}$ 1971. Print.

Cantarella, Eva. ,Revenge and Punishment.” Ancient Greek Law in the $21^{\text {st }}$ Century. Hg. Paula Perlman. Austin: U of Texas P, 2018. 25-34.

Campe, Rüdiger und Julia Weber. „Rethinking Emotion: Moving beyond Interiority.“ Rethinking Emotion: Interiority and Exteriority in Premodern, Modern, and Contemporary Thought. Berlin: de Gruyter, 2014. 1-20. Print.

Dawson, Lesel und Fiona McHardy. Revenge and Gender in Classical, Medieval and Renaissance Literature. Edinburgh: Edinburgh UP, 2018. Print.

Descharmes, Bernadette. Rächer und Gerächte. Konzeption, Praktiken und Loyalitäten der Rache im Spiegel der attischen Tragödie. Göttingen: V\&R, 2013. Print.

Durkheim, Émile. Les formes élémentaires de la vie religieuse. Le système totémique en Australie. Paris: Librairie Générale Française, 1991. Print.

Ermers, Robert. Honor Related Violence: A New Social Psychological Perspective. London: Routledge, 2018. Print.

Ette, Wolfram. „Trauer und Trauerspiel. Spätantike, Frühe Neuzeit, Moderne.“ Handbuch Literatur und Emotionen. Hg. Martin von Koppenfels und Cornelia Zumbusch. Berlin: de Gruyter, 2016. 290-312. Print.

Freud, Sigmund. [GW] Gesammelte Werke. Chronologisch geordnet. Hg. Anna Freud u.a. Frankfurt am Main: Fischer, 1968. Print.

Greiner, Bernhard. „Constructions of Interiority around 1800.“ Rethinking Emotion: Interiority and Exteriority in Premodern, Modern, and Contemporary Thought. Berlin: de Gruyter, 2014. 137-170. Print. 
Grimm, Jacob. Deutsche Rechtsalterthümer. Göttingen: Diederichsche Buchhandlung, 1828. Print.

Grimm, Jacob und Wilhelm. [DW] Deutsches Wörterbuch. Nachdruck der Erstausg. 1878ff. München: dtv, 1984. Print.

Groves, James E. Hamlet on the Couch: What Shakespeare Taught Freud. London: Routledge, 2018. Print.

Heidegger, Martin. Grundbegriffe der aristotelischen Philosophie. [GA] Gesamtausgabe. Frankfurt am Main: Klostermann, 1976ff. Bd. 18. Print.

Jacoby, Susan. Wild Justice: The Evolution of Revenge. New York, NY: Harper \& Row, 1983. Print.

Kaufmann, E. „Rache“. Handwörterbuch zur Deutschen Rechtsgeschichte. 25. Lieferung. Hg. Adalbert Erler u.a. Berlin: Erich Schmidt, 1985. 126-127. Print.

Kerrigan, John. Revenge Tragedy: Aeschylus to Armageddon. Oxford: Claredon, 1996. Print.

Lehmann, Johannes F. „Geschichte der Gefühle. Wissensgeschichte, Begriffsgeschichte, Diskursgeschichte." Handbuch Literatur und Emotionen. Hg. Martin von Koppenfels und Cornelia Zumbusch. Berlin: de Gruyter, 2016. 140-157. Print.

Nietzsche, Friedrich. Zur Genealogie der Moral. Eine Streitschrift. Kritische Studienausgabe 5. Hg. Giorgio Colli und Mazzino Montinari. München: dtv, 1980. Print.

Nussbaum, Martha. Anger and Forgiveness: Resentment, Generosity, Justice. Oxford: Oxford UP, 2016. Print.

Probst, Peter und Gerhard Sprenger. „Rache“. Historisches Wörterbuch der Philosophie. Hg. Joachim Ritter u.a. Basel: Schwabe, 2017. Web. [16. August 2018]

Richter, Simon. Laocoon's Body and the Aesthetics of Pain: Winckelmann, Lessing, Herder, Moritz, Goethe. Detroit: Wayne State UP, 1992. Print.

Segesser, Daniel M. Recht statt Rache oder Rache durch Recht? Die Ahndung von Kriegsverbrechen in der internationalen fachwissenschaftlichen Debatte 1872-1945. Paderborn: Schöningh, 2007. Print.

Stepanova, Maria. Nach dem Gedächtnis. Übers. Olga Radetzkaja. Berlin: Suhrkamp, 2018.

Speziale-Bagliacca, Roberto. Guilt: Revenge, Remorse and Responsibility after Freud. Hove: Brunner-Routledge, 2004. Print.

Weissberg, Liliane. „Language's Wound: Herder, Philoctetes, and the Origin of Speech”. MLN 104 (1989): 548-579. Print.

Wiggins, Kyle (Hg.) American Revenge Narratives: A Collection of Critical Essays. Baskingstoke: Palgrave Macmillan, 2018. Print.

Yoshimura, Steven M. and Susan D. Boon. Communicating Revenge in Interpersonal Relationships. Lanham: Lexington, 2018. Print.

Zaibert, Leo. Rethinking Punishment. Cambridge: Cambridge UP, 2018. Print.

Juliane Prade-Weiss

Yale University

Department of German

P.O. Box 208210

New Haven, CT 06520-8210, USA

prade@lingua.uni-frankfurt.de
Jens Klenner

Bowdoin College

Department of German

7700 College Station

Brunswick, ME 04011, USA

jklenner@bowdoin.edu 